Article

\title{
Computer-Aided Discovery of New Solubility-Enhancing Drug Delivery System
}

\author{
Mikołaj Mizera ${ }^{1,2}\left({ }^{\oplus}\right.$, Eugene N. Muratov ${ }^{2,3}{ }^{\oplus}$, Vinicius M. Alves ${ }^{2}$, Alexander Tropsha ${ }^{2, *}$ and \\ Judyta Cielecka-Piontek 1,*(D) \\ 1 Department of Pharmacognosy, Faculty of Pharmacy, Poznań University of Medical Sciences, Święcickiego 4, \\ 60-781 Poznań, Poland; mikolajmizera@gmail.com \\ 2 Laboratory for Molecular Modeling, Division of Chemical Biology and Medicinal Chemistry, UNC Eshelman \\ School of Pharmacy, University of North Carolina, Chapel Hill, NC 27599, USA; \\ murik@email.unc.edu (E.N.M.); alvesv@email.unc.edu (V.M.A.) \\ 3 Department of Pharmaceutical Sciences, Federal University of Paraíba, Joao Pessoa 58059, PB, Brazil \\ * Correspondence: alex_tropsha@unc.edu (A.T.); jpiontek@ump.edu.pl (J.C.-P.)
}

Received: 5 May 2020; Accepted: 14 June 2020; Published: 16 June 2020

check for updates

\begin{abstract}
The poor aqueous solubility of active pharmaceutical ingredients (APIs) places a limit on their therapeutic potential. Cyclodextrins (CDs) have been shown to improve the solubility of APIs, but the magnitude of the improvement depends on the structure of both the CDs and APIs. We have developed quantitative structure-property relationship (QSPR) models that predict the stability of the complexes formed by a popular poorly soluble antibiotic, cefuroxime axetil (CA) and different CDs. We applied this model to five CA-CD systems not included in the modeling set. Two out of three systems predicted to have poor stability and poor CA solubility, and both CA-CD systems predicted to have high stability and high CA solubility were confirmed experimentally. One of the CDs that significantly improved CA solubility, methyl- $\beta C D$, is described here for the first time, and we propose this $\mathrm{CD}$ as a novel promising excipient. Computational approaches and models developed and validated in this study could help accelerate the development of multifunctional CDs-based formulations.
\end{abstract}

Keywords: quantitative structure-property relationship; cyclodextrins; cefuroxime axetil

\section{Introduction}

Poor solubility of active pharmaceutical substances (API) places a significant limitation on their clinical use. This issue affects nearly $40 \%$ of currently marketed APIs, resulting in their low bioavailability and the necessity of increased API doses. Unfortunately, this issue is persistent: it has been estimated that $90 \%$ of APIs currently under development are poorly soluble [1,2]. APIs with low solubility belong to groups II and IV in the Biopharmaceutics Classification System (BCS). BCS II substances are poorly soluble and have high permeability, whereas group IV substances are poorly soluble and have low permeability. Optimizing pharmaceutical formulations toward greater solubility may substantially improve their bioavailability.

The use of solubilizers as excipients allows for a compromise between increasing the concentration of APIs at the site of release and maintaining the lipophilic nature of the APIs that facilitates their permeability [3]. Among many strategies for combating poor solubility, the use of solubilizing excipients such as cyclodextrins (CDs) is one of the most popular ones. CDs are macrocyclic polymers that can encapsulate API molecules within the lipophilic cavity or adhere to API at the hydrophilic surface. CDs have been successfully used as solubilizers for many sparingly soluble APIs [4-6]. Multiple examples have demonstrated the plausible effect of CDs on the chemical stability of APIs [7], 
including the pronounced stabilizing effect on $\beta$-lactam antibiotics in an acidic environment [8]. The stabilizing effect of CDs on the crystal structure [9] is crucial for preserving the required dissolution rate throughout the shelf period of drugs, such as cefuroxime axetil (CA) [10]. The separation of diastereomers, affecting the bioactivity of CA, may be achieved by the application of CDs [11]. CDs as multifunctional excipients may be also used for taste masking [12-14], which is especially important to improve the comfort of patients taking bitter drugs such as $\beta$-lactam antibiotics.

The vast number of experimental studies on CDs as multifunctional excipients has contributed to the availability of large data sets with known thermodynamic properties of API-CD systems, including synthetic CDs approved by FDA/EMA for use in pharmaceutical development or as food additives. Indeed, many poorly soluble APIs have entered the pharmaceutical market as a result of using CDs for drug formulations [15]. Many observations indicating that the solubilizing effect of CDs on APIs depends on the structures of both components have made the API-CD systems a good target for quantitative structure-property relationship (QSPR) modeling. If successful, such models could be very useful in the rational design of effective formulations.

The interaction of APIs with CDs can be characterized by the stability constant $K_{s}$ [7], which is related to change of API solubility $\Delta S$ according to the formula:

$$
\Delta S=\frac{K_{S} S_{0}}{1+K_{s} S_{0}}[C D]
$$

where:

$S_{0}$-intrinsic solubility of API,

$\mathrm{K}_{\mathrm{s}}$-stability constant of API-CD system,

$[C D]-C D$ concentration.

Machine learning-based QSPR models have shown good performance in predicting $K_{s}$. For instance, Jeschke et al. [16] developed a model for $K_{s}$ prediction of $\beta C D$ systems. Their model employed data on consistently measured $\Delta G$ of formation for $\beta C D$ systems, and the random forest method achieving the external validation $\mathrm{R}^{2}=0.66$. Zhao et al. [17] compared gradient boosting and deep neural network models for predicting $\Delta G$ of complex formation. Gradient boosting showed good predictive performance indicated by consistent in-sample and out-of-sample scores $\mathrm{R}^{2}=0.86$, while DNN resulted in a model with $R^{2}=0.76$ and $R^{2}=0.62$ for in-sample and out-of-sample predictions, respectively. A computer-aided study resulting in the synthesis of a novel polymer drug carrier system was performed by Alves et al. [18]. The model used descriptors of both API and polymer to streamline the selection of the most promising carrier system for poorly soluble APIs. The predictions were experimentally validated and led to the discovery of a new solubility enhancing carrier system.

Herein, we have investigated the use of optimal $C D$ to improve the solubility and bioavailability of $\mathrm{CA}$, a sparingly soluble $\beta$-lactam analog. Although the increased solubility of CA in an amorphous form was shown [19], some limitations related to recrystallization during shelf time apply. The possible application of CDs to stabilize the amorphous state of solid CA may be beneficial for achieving higher stability and better solubility [20]. Furthermore, improved compliance may be achieved by masking the extremely bitter taste of CA [21].

In the previous studies, we investigated the benefits of combining CA with $C D$ in the solid phase in terms of improving CA's solubility and antimicrobial activity [22]. Herein, we have carried out the QSPR modeling of $K_{s}$. The main objective of this study was computer-assisted identification of stable CA-CD systems with increased solubility of CA. To achieve this goal, we executed the following specific studies: (i) collection, curation and integration of publicly available data on the stability of $C D$ complexes with small molecules; (ii) development of novel descriptors of API-CD systems; (iii) QSPR model development and validation, (iv) prediction of $\ln \left(K_{s}\right)$ for novel CA-CDs complexes for CDs in our in-house collection and (v) experimental validation of computational predictions. 


\section{Materials and Methods}

\subsection{Data Integration and Curation}

Data used in the study were acquired from the Cyclodextrin knowledgebase [23]. The data reported in the database contained the IUPAC name of API, IUPAC name of CD, the value of the stability constant and the experimental conditions of stability constant evaluation if reported in the source study. The structure of molecules was generated using OPSIN [24] and ChemAxon [25] IUPAC name converters, and subsequently standardized using ChemAxon Standardizer [26] following the protocols we developed previously $[27,28]$. Chirality information was preserved during structure generation from the IUPAC names. The curated dataset is available as supplemental material (File S1).

\subsection{Molecular Descriptors}

Descriptors for APIs structures were calculated using Mordred [29], which implemented PaDEL descriptors in Python. 3D descriptors were used, since chirality information was present in provided structures. A total of 637 descriptors were successfully generated and used for model development. A novel protocol for calculating CD descriptors was developed. The main macrocyclic ring was described by a single number of glucose subunits and concatenated with Mordred descriptors of the C -5 side chain terminated by a hydrogen atom. This procedure allowed for the increased variance in descriptor space due to the separation of a similar macrocyclic ring from side chains.

\subsection{Model Development and Validation}

The gradient boosting algorithm was used to create the QSPR model. The basis of operation of the algorithm is an iterative addition of decision trees to an ensemble to reduce the error of the previous trees in this ensemble. The specific implementation used in our modelling study was LightGBM library [30]. The model was validated using a 5-fold external cross-validation procedure [31]. The procedure involves a random division of the entire dataset into five subsets of nearly equal size followed by the systematic binary division of the original dataset into a training set with $80 \%$ of all samples used for model training and $20 \%$ of the samples used as a test set, such that each of the five subsets would be used once as a test set. For each 80:20 division, a new model was trained and used for the prediction of the test set. The test predictions were collected and used for statistical characteristics calculation. For the final model, a 5-fold cross-validation grid-search was done on the whole dataset, and the best model was refitted on the whole dataset before predicting $\ln \left(K_{s}\right)$ of the CA-CD systems.

\subsection{Statistical Analysis}

Predictive performance evaluation of the model was done by calculation of the following measures: accuracy, area under the receiver operating characteristic curve (AUC), correct classification rate (CCR), sensitivity, positive predictive value (PPV), specificity and negative predictive value (NPV) [18].

\subsection{Systems Stability Prediction and Experimental Evaluation}

A poorly soluble API from the BCS II class, CA was selected as a model compound for which the best CD expected to improve CA solubility was predicted. The CD library consisted of an in-house collection of CDs commonly used in pharmaceutical formulations, including $\alpha C D, \beta C D, H P-\alpha C D, H P-\beta C D$ and methyl- $\beta C D$. For each CA-CD system, predictions were evaluated experimentally. Stability constants of CA-CD systems were measured using the phase-solubility technique [6]. The excess of CA was added to a $15 \mathrm{~mL}$ of CD solutions in concentrations of $0.02-0.1 \mathrm{mmol}$ in stoppered test tubes. The test tubes were shaken on a rotary shaker for $72 \mathrm{~h}$ at a controlled temperature $25^{\circ} \mathrm{C}$ and $\mathrm{pH}=7$. The samples were filtered by a $25 \mu \mathrm{m}$ filter and the concentration of CA was measured using UV spectroscopy at $281 \mathrm{~nm}$ with the PerkinElmer Lambda 35 UV/Vis Spectrometer. 


\section{Results}

\subsection{Study Design}

The study design is shown in Figure 1. Data on the stability of multiple small molecules-CD systems characterized by their $K_{s}$ were collected from publicly accessible sources, as described in detail in Methods (Figure 1, Step 1). The data were curated (Figure 1, Step 2), and the reported experimental values were converted to $\ln \left(K_{s}\right)$. Small molecule-CD systems were characterized by standard descriptors and a novel protocol for CD descriptor generation was used (Figure 1, Step 3). QSPR models were developed with a gradient boosting machine learning approach (Figure 1, Step 4). Models were employed to predict the stability of CA-CD systems for five CDs from our in-house collection, as described in Methods (Figure 1, Step 5), and all five systems were tested experimentally (Figure 1, Step 6).

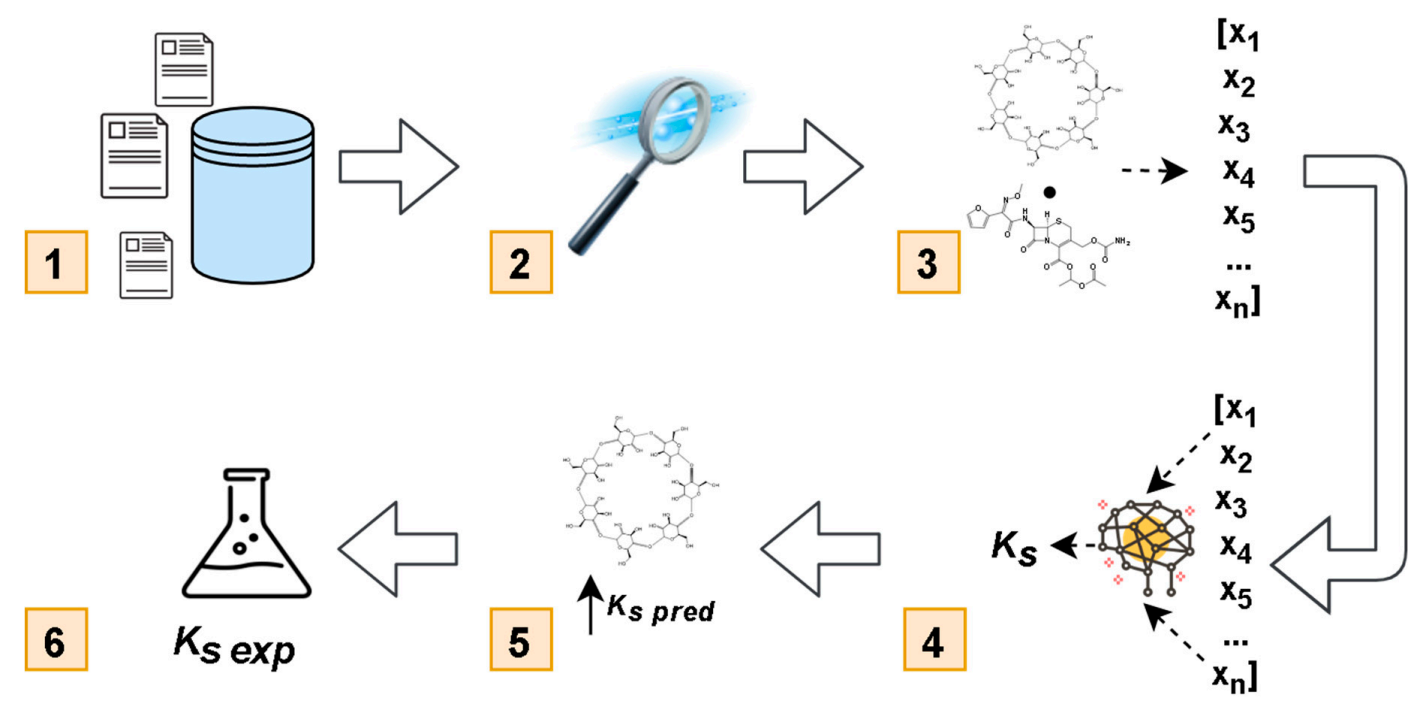

Figure 1. Study design: data collection (1); data curation (2); descriptors calculation (3); model development (4); $\ln \left(K_{s}\right)$ prediction (5); experimental validation (6).

\subsection{Dataset Preparation}

We employed data reported in the database of CD complexes with small molecules [23] that initially included 8534 records (Figure 2, Step 1). These data were curated based on the protocols described by us earlier $[27,28]$. Records without structural information (131 in total) were removed (Figure 2, Step 2). These included records for compounds for which neither SMILES nor IUPAC names were provided. For the remaining compounds, their IUPAC names were converted to 2D chemical structures by ChemAxon software [25] (Figure 2, Step 3) or manually in the case of failure of the automated procedure. Sixty-three records with IUPAC names that could not be associated with molecular structure were removed along with single-atom counter-ions (Figure 2, Step 4). All records included data on the experimental conditions such as the temperature and $\mathrm{pH}$; for records with missing experimental conditions, the normal conditions, i.e., $\mathrm{T}=25^{\circ} \mathrm{C}$ and $\mathrm{pH}=7$, were assumed. Records in the dataset were considered duplicative if both $\mathrm{InChI}$ keys of their small molecules and corresponding CDs in the system, as well as experimental conditions, were identical. If the difference between $\ln \left(K_{s}\right)$ values for duplicate small molecule-CD systems under the same experimental conditions was less than $10 \%$ of the range of $\ln \left(K_{s}\right)$ values, duplicative records were merged, and the averaged $\ln \left(K_{s}\right)$ value was assigned to the merged record; otherwise, all duplicated records were removed (Figure 2, Step 5). This step resulted in 418 merged records and 4540 records that were removed. The structures of small molecules with the molecular weight greater than 50 Da and less than 500 Da were kept (Figure 2, Step 6). The execution of Step 6 resulted in removing 183 records. 


\begin{tabular}{|c|c|c|}
\hline & & atapoints \\
\hline 1 & Collected data & 8534 \\
\hline 2 & No structural information & 8403 \\
\hline 3 & IUPAC to $2 \mathrm{D}$ structure & 8403 \\
\hline 4 & Failed IUPAC to structure & 8340 \\
\hline 5 & Duplicates analysis & 3359 \\
\hline 6 & Molecular weight & 3176 \\
\hline 7 & Experimental conditions & 1812 \\
\hline 8 & Outliers analisys & 1654 \\
\hline & Final dataset & 1654 \\
\hline
\end{tabular}

Figure 2. Data curation workflow. Number of data points shown in each row represent the number of compounds left after the respective curation step.

Records with reported temperature and $\mathrm{pH}$ close to our test conditions $\mathrm{T}=25^{\circ} \mathrm{C}$ and $\mathrm{pH}=7$ were kept. Therefore, we selected only data from the experiments carried out under temperatures between $20^{\circ} \mathrm{C}$ and $30^{\circ} \mathrm{C}$ and pH between 5 and 8 (Figure 2, Step 7). The final dataset included 1654 small molecule-CD complexes with $\ln \left(K_{s}\right)$ values ranging from $\ln \left(K_{s}\right)=2$ to $\ln \left(K_{s}\right)=10$, where the datapoints outside these boundaries were considered outliers (Figure 2, Step 8). The distribution of CD types in the final dataset is shown in Table 1. The dataset is dominated by natural CDs: $\alpha, \beta$ and $\gamma$. Of the underrepresented class of semisynthetic CDs, the representatives with the highest counts were hydroxypropyl- $\beta C D$ (HP- $\beta C D$ ) and methylated derivatives of $\beta C D$ s (Dimethyl- $\beta C D$, randomly methylated- $\beta C D$, Trimethyl $\beta C D$ ). The entire dataset is included in the Supplemental Materials (S1).

Table 1. Distribution of cyclodextrin (CD) derivatives in the final dataset.

\begin{tabular}{|c|c|c|c|c|c|}
\hline \multicolumn{2}{|c|}{$\alpha \mathrm{CD}$ and Derivatives } & \multicolumn{2}{|c|}{$\beta C D$ and Derivatives } & \multicolumn{2}{|c|}{$\gamma \mathrm{CD}$ and Derivatives } \\
\hline Type & Samples & Type & Samples & Type & Samples \\
\hline$\alpha \mathrm{CD}$ & 411 & Acetyl- $\beta C D$ & 19 & $\gamma \mathrm{CD}$ & 160 \\
\hline Carboxyl $\alpha \mathrm{CD}$ & 1 & $\beta C D$ & 638 & Hydroxypropyl- $\gamma C D$ & 5 \\
\hline Hydroxypropyl- $\alpha \mathrm{CD}$ & 3 & Carboxyl- $\beta C D$ & 15 & & \\
\hline \multirow[t]{7}{*}{ Trimethyl- $\alpha \mathrm{CD}$} & 8 & Dimethyl- $\beta C D$ & 52 & & \\
\hline & & Hydroxypropyl- $\beta C D$ & 136 & & \\
\hline & & Randomly & 49 & & \\
\hline & & $\begin{array}{l}\text { med sulfate } \\
\beta C D\end{array}$ & 16 & & \\
\hline & & Sulfobutyl ether $\beta C D$ & 117 & & \\
\hline & & Succinate- $\beta C D$ & 5 & & \\
\hline & & Trimethyl- $\beta C D$ & 19 & & \\
\hline
\end{tabular}

\subsection{Model Development}

The data used for model development showed normal distribution with the mean $\ln \left(K_{s}\right)=5.37$ and standard deviation $=2.22$ (Figure 3 ). The regression model we built initially failed to achieve acceptable statistical characteristics. A possible cause for the failure was the relatively large fraction 
of the data with missing experimental conditions and the inadequacy of our guessed conditions for building a successful regression model. Therefore, we decided to switch from the regression approach to the more approximate binary classification. The final model consisted of three binary models developed for three different binary division thresholds of $\ln \left(K_{s}\right): 4.5$ (Figure 3, red), 5 (Figure 3, gray) and 5.5 (Figure 3, green). The selection of thresholds for different binary classifications was done based on the range of reported stability constants of $\ln \left(K_{s}\right)$ for CA-CD systems [32,33]. For negative classes, the values within 1.5 logarithmic units below the chosen threshold were used (Figure 3, dotted lines). The positive values included data above the threshold; the exact upper boundary was adjusted to achieve an approximate match between the number of positive samples and that of the negative samples. The classification dataset with the threshold of 4.5 included a total of 1145 samples, with 584 positives and 561 negatives (Figure 3, red); for classification with the threshold 5, the dataset included a total of 1316 samples, comprising 671 positives and 645 negatives (Figure 3, gray); and for classification with threshold 5.5, the dataset included 1460 samples: 745 positives and 715 negatives (Figure 3, green). Models were trained and tested using the five-fold external cross-validation procedure, and the statistical characteristics of the models are given in Table 2. The model achieved acceptable scores for all main statistical characteristics according to the suggested best practices for QSAR models validation [34].

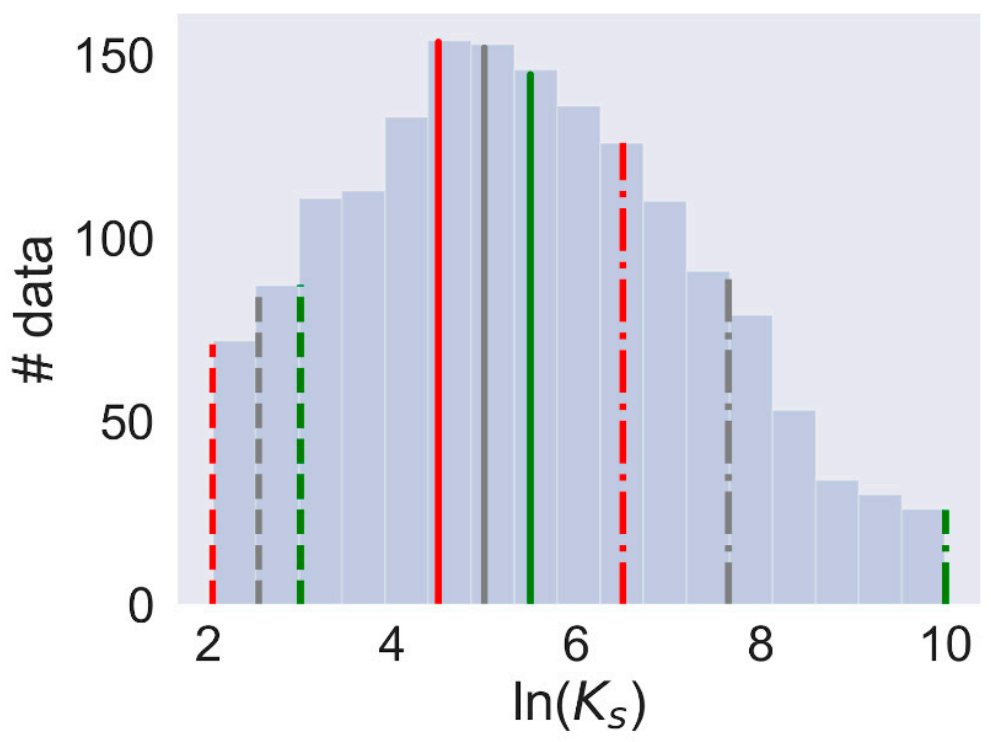

Figure 3. Distribution of $\ln \left(K_{s}\right)$ values in the dataset. Thresholds for binary classification models: 4.5 (red), 5.0 (gray), and 5.5 (green).

Table 2. Statistical characteristics. The characteristics of the models for 5-fold external cross-validation.

\begin{tabular}{cccccccc}
\hline Class & Accuracy & AUC & CCR & Sensitivity & PPV & Specificity & NPV \\
\hline $\ln \left(K_{s}\right) \geq 4.50$ & 0.64 & 0.69 & 0.64 & 0.68 & 0.63 & 0.60 & 0.65 \\
$\ln \left(K_{s}\right)>5.00$ & 0.67 & 0.75 & 0.67 & 0.71 & 0.66 & 0.64 & 0.69 \\
$\ln \left(K_{s}\right)>5.50$ & 0.70 & 0.76 & 0.70 & 0.69 & 0.67 & 0.71 & 0.72 \\
\hline
\end{tabular}

\subsection{Prediction and Experimental Validation}

Model-based predictions in comparison with the experimental results are summarized in Table 3. Systems were considered promising if all three models predicted a CD-CA system as positive. According to this protocol, $C A-\beta C D$ and $C A-$ methyl- $\beta C D$ were selected as promising hits, and all classification predictions for these two systems were confirmed experimentally (Table 3, Systems 2 and 5). The CA- $\beta C D$ system was investigated by Sapte et al. [33] before our study, and $\ln \left(K_{s}\right)=5.83$ was reported for this system. However, we decided to exclude this record from our training database, and use it for an additional external validation of both the experimental data collected in our laboratory 
and the model we developed. Indeed, the $C A-\beta C D$ system was predicted correctly as highly stable, and our experimental value of $\ln \left(K_{s}\right)=5.72$ also agreed with the literature data. To examine the predictive performance of the models further, the three systems selected as not promising (Table 3, Systems 1, 3 and 4) were also tested. The experimental results for these systems were in full agreement with predictions for $C A-\alpha C D$ and $C A-H P-\alpha C D$ and in partial agreement for CA-HP- $\beta C D$. The latter system showed high $\ln \left(K_{s}\right)$, which was correctly predicted by two out of three models. In total, out of 15 predictions for all tested systems, 14 (93.3\%) were correct; i.e., the $\ln \left(K_{s}\right)$ value for the CA-HP- $\beta C D$ system was underestimated in one case (Table 3, System 4). Results obtained in our laboratory were in agreement with the value $\ln \left(K_{s}\right)=5.95$ reported by Shah et al. [32] (Table 3, System 4) confirming the compatibility of our measurements with the data reported in the independent literature.

Table 3. Results of experimental validation. Comparison of predicted versus actual stability classes for different cefuroxime axetil (CA)-CD systems and different $\ln \left(K_{s}\right)$ thresholds for binary data division. Measured experimental $\ln \left(K_{s}\right)$ values are shown in the last column of the table.

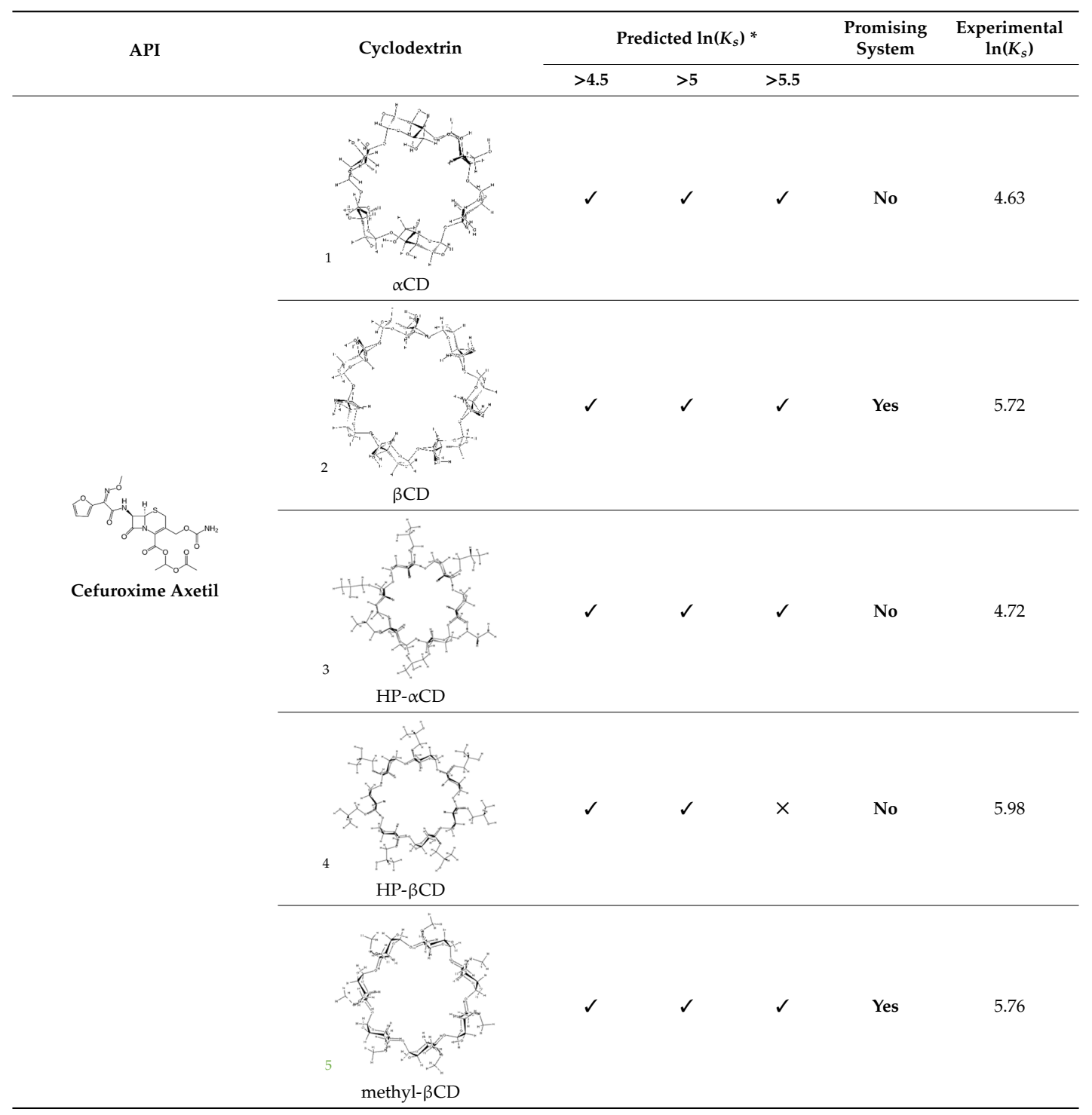

* Accurate prediction is labeled as $(\checkmark)$ while inaccurate is labeled as $(\times)$. 


\section{Discussion}

The first goal of the study was to collect and curate data required for the machine-learning-based QSPR model. The curated dataset consisted of diverse API and non-API chemical structures in systems with 16 CDs of natural and semi-synthetic origin. Previously, to model guest-CD systems, an effort was made to curate the data originating from the distinct literature studies [35,36], including a large library of both guest molecules and CDs [17]. We have followed the best practices of QSAR modeling [31] to perform our study and assure of its reproducibility.

Initially, we developed continuous QSAR models that failed to achieve acceptable statistical characteristics, i.e., the $\mathrm{Q}^{2}$ was $<0.5$. Thus, we decided to employ binary classification models and developed three models with cut-off thresholds of 4.5, 5.0 and 5.5, respectively. To develop these models, we employed the gradient boosting algorithm and chemical descriptors reflecting the structure of both APIs and side chains of CDs. The size of the CD macrocycles was described by the number of constituting glucose residues. Due to the enantioselectivity of CDs [37], the information on API chiral centers was preserved by using 3D descriptors. One of the biggest advantages of this model is that it could be applied to a variety of CDs and experimental conditions instead of being specific for a single $\mathrm{CD}$ and predefined temperature and $\mathrm{pH}[35,38]$. Consideration of both guest and CD structures makes the model useful in cases when no sufficient experimental data for a CD system exist. This problem is especially common when synthetic derivatives of CDs are used. The predictive power of all the models was rigorously validated using 5-fold external cross-validation (see Table 2 for statistical characteristics).

To achieve the goal of this study, we have applied the developed QSPR models to the prediction of the stability constant for a poorly soluble $\beta$-lactam antibiotic, $C A$, combined with five CDs from our in-house collection. We were particularly interested in combinations that would be predicted as positives by all three models as an indication of the higher confidence in the positive result. For all tested systems, 14 out of 15 predictions were correct. The predictions were used to identify the system with likely the highest $\ln \left(K_{s}\right)$. Two CA-CD systems ( 2 and 5 from Table 3 ) were selected as the most promising, because the models predicted these systems to have high $\ln \left(K_{s}\right)$ values; three other systems were expected to have lower $\ln \left(K_{s}\right)$ values. Both systems predicted to have high $\ln \left(K_{s}\right)$ were confirmed successfully with a minor exception for the CA-HP- $\beta C D$ system ( 4 in Table 3 ), and correct predictions were made by two out of three models. One of the models with the highest $\ln \left(K_{s}\right)$ threshold for binary division of the training set of $\ln \left(K_{S}\right)=5.5$ mis-predicted this compound as negative, whereas this system possessed the highest experimental $\ln \left(K_{S}\right)$ values. As the most important outcome of our study, the identified CA-methyl- $\beta C D$ (5 in Table 3) is a novel, not previously reported CD system, which may serve as a promising excipient in drug formulation with CA.

Moreover, CD systems identified in this work may be of additional value for diastereomers separation [11], solid-state chemical stabilization under certain conditions [7] and bitter taste masking $[12,13]$.

\section{Conclusions}

To summarize, we have successfully developed predictive models for assessing the stability of a broad range of API-CD systems. Using these models, we have identified and experimentally confirmed a novel promising CA-methyl- $\beta C D$ system with improved CA solubility. This success was enabled by the careful study design, including the collection of the expansive and diverse training dataset, thorough data curation and the use of the best practices of QSAR modeling, including rigorous external validation of the models. The successful experimental validation of the developed models using poorly soluble API such as CA proved the applicability of the model to the discovery of novel API-CD systems, where the solubility of the API can be substantially improved by the application of natural and semi-synthetic CDs. The range of this model's application can be extended to streamlining the choice of CDs for the pharmaceutical analysis of chiral compounds [37,39] and the phytochemical extraction 
of APIs from plant matrix [40]. In both applications, a high affinity of CDs to the desired molecules may be used to improve the efficiency of the analytical method, or reduce the toxic solvents use.

Supplementary Materials: The following are available online at http://www.mdpi.com/2218-273X/10/6/913/s1, File S1: Dataset used for model development.

Author Contributions: Conceptualization, M.M., E.N.M., A.T. and J.C.-P.; data curation, M.M.; formal analysis, E.N.M., A.T. and J.C.-P.; funding acquisition, M.M. and J.C.-P.; investigation, M.M.; methodology, M.M., E.N.M. and V.M.A.; project administration, J.C.-P.; resources, J.C.-P.; software, M.M.; supervision, E.N.M. and A.T.; validation, M.M.; visualization, M.M.; writing—original draft, M.M.; writing—review and editing, E.N.M., A.T. and J.C.-P. All authors have read and agreed to the published version of the manuscript.

Funding: This research was funded Ministry of Science and Higher Education, grant number DI2014020544; and the $\mathrm{NIH}$, grant number [5U54CA198999]. This research was also supported in part by the PL-Grid Infrastructure. M.M. acknowledges the doctoral scholarship support from the National Science Center DEC-2018/28/T/NZ7/00472.

Conflicts of Interest: The authors declare no conflict of interest.

\section{References}

1. Loftsson, T.; Brewster, M.E. Pharmaceutical applications of cyclodextrins: Basic science and product development. J. Pharm. Pharmacol. 2010, 62, 1607-1621. [CrossRef]

2. GlobalData Healthcare. CPHI Experts: $90 \%$ of Current Pipeline APIs Are Poorly Soluble-Pharmaceutical Technology. Available online: https:/www.pharmaceutical-technology.com/comment/cphi-experts-90current-pipeline-apis-poorly-soluble/ (accessed on 16 June 2020).

3. Fine-Shamir, N.; Beig, A.; Zur, M.; Lindley, D.; Miller, J.M.; Dahan, A. Toward Successful Cyclodextrin Based Solubility-Enabling Formulations for Oral Delivery of Lipophilic Drugs: Solubility-Permeability Trade-Off, Biorelevant Dissolution, and the Unstirred Water Layer. Mol. Pharm. 2017, 14, 2138-2146. [CrossRef]

4. Adeoye, O.; Conceição, J.; Serra, P.A.; Bento da Silva, A.; Duarte, N.; Guedes, R.C.; Corvo, M.C.; Aguiar-Ricardo, A.; Jicsinszky, L.; Casimiro, T.; et al. Cyclodextrin solubilization and complexation of antiretroviral drug lopinavir: In silico prediction; Effects of derivatization, molar ratio and preparation method. Carbohydr. Polym. 2020, 227, 115287. [CrossRef]

5. Jansook, P.; Kulsirachote, P.; Loftsson, T. Cyclodextrin solubilization of celecoxib: Solid and solution state characterization. J. Incl. Phenom. Macrocycl. Chem. 2018, 90, 75-88. [CrossRef]

6. Saokham, P.; Muankaew, C.; Jansook, P.; Loftsson, T. Solubility of Cyclodextrins and Drug/Cyclodextrin Complexes. Molecules 2018, 23, 1161. [CrossRef]

7. Popielec, A.; Loftsson, T. Effects of cyclodextrins on the chemical stability of drugs. Int. J. Pharm. 2017, 531, 532-542. [CrossRef] [PubMed]

8. Popielec, A.; Fenyvesi, É.; Yannakopoulou, K.; Loftsson, T. Effect of cyclodextrins on the degradation rate of benzylpenicillin. Pharmazie 2016, 71, 68-75. [PubMed]

9. Iohara, D.; Anraku, M.; Uekama, K.; Hirayama, F. Modification of Drug Crystallization by Cyclodextrins in Pre-formulation Study. Chem. Pharm. Bull. 2019, 67, 915-920. [CrossRef] [PubMed]

10. Talaczynska, A.; Mizera, M.; Szybowicz, M.; Nowicka, A.B.; Garbacki, P.; Paczkowska, M.; Zalewski, P.; Kozak, M.; Oszczapowicz, I.; Jelinska, A.; et al. Studies of the crystalline form of cefuroxime axetil: Implications for its compatibility with excipients. Acta Pol. Pharm. 2016, 73, 1299-1309. [PubMed]

11. Delplanques, T.; Boulahjar, R.; Charton, J.; Houze, C.; Howsam, M.; Servais, A.-C.; Fillet, M.; Lipka, E. Single and dual cyclodextrins systems for the enantiomeric and diastereoisomeric separations of structurally related dihydropyridone analogues. Electrophoresis 2017, 38, 1922-1931. [CrossRef] [PubMed]

12. Lenik, J.; Wesoły, M.; Ciosek, P.; Wróblewski, W. Evaluation of taste masking effect of diclofenac using sweeteners and cyclodextrin by a potentiometric electronic tongue. J. Electroanal. Chem. 2016, 780, 153-159. [CrossRef]

13. Liu, T.; Wan, X.; Luo, Z.; Liu, C.; Quan, P.; Cun, D.; Fang, L. A donepezil/cyclodextrin complexation orodispersible film: Effect of cyclodextrin on taste-masking based on dynamic process and in vivo drug absorption. Asian J. Pharm. Sci. 2019, 14, 183-192. [CrossRef] [PubMed]

14. Chay, S.K.; Keating, A.V.; James, C.; Aliev, A.E.; Haider, S.; Craig, D.Q.M. Evaluation of the taste-masking effects of (2-hydroxypropyl)- $\beta$-cyclodextrin on ranitidine hydrochloride; a combined biosensor, spectroscopic and molecular modelling assessment. RSC Adv. 2018, 8, 3564-3573. [CrossRef] 
15. Jansook, P.; Ogawa, N.; Loftsson, T. Cyclodextrins: Structure, physicochemical properties and pharmaceutical applications. Int. J. Pharm. 2018, 535, 272-284. [CrossRef] [PubMed]

16. Jeschke, S.; Cole, I.S. 3D-QSAR for binding constants of $\beta$-cyclodextrin host-guest complexes by utilising spectrophores as molecular descriptors. Chemosphere 2019, 225, 135-138. [CrossRef]

17. Zhao, Q.; Ye, Z.; Su, Y.; Ouyang, D. Predicting complexation performance between cyclodextrins and guest molecules by integrated machine learning and molecular modeling techniques. Acta Pharm. Sin. B 2019, 9, 1241-1252. [CrossRef]

18. Alves, V.M.; Hwang, D.; Muratov, E.; Sokolsky-Papkov, M.; Varlamova, E.; Vinod, N.; Lim, C.; Andrade, C.H.; Tropsha, A.; Kabanov, A. Cheminformatics-driven discovery of polymeric micelle formulations for poorly soluble drugs. Sci. Adv. 2019, 5, eaav9784. [CrossRef]

19. Oszczapowicz, I.; Małafiej, E.; Szelachowska, M.; Horoszewicz-Małafiej, A.; Kuklewicz, C.; Sierańska, E.; Denys, A.; Niedworok, J. Esters of cephalosporins. Part II. Differences in the properties of various forms of the 1-acetoxyethyl ester of cefuroxime. Acta Pol. Pharm. 1995, 52, 397-401.

20. Dalal, N.; Buckner, I.S.; Wildfong, P.L.D. Experimental Determination and Theoretical Calculation of the Eutectic Composition of Cefuroxime Axetil Diastereomers. AAPS PharmSciTech 2017, 18, 2570-2578. [CrossRef]

21. Du, Y.; Zhai, Y.; Zhang, J.; Wu, C.; Luo, C.; Sun, J.; He, Z. Development and evaluation of taste-masked dry suspension of cefuroxime axetil for enhancement of oral bioavailability. Asian J. Pharm. Sci. 2013, 8 , 287-294. [CrossRef]

22. Mizera, M.; Szymanowska, D.; Stasiłowicz, A.; Siąkowska, D.; Lewandowska, K.; Miklaszewski, A.; Plech, T.; Tykarska, E.; Cielecka-Piontek, J. Computer-Aided Design of Cefuroxime Axetil/Cyclodextrin System with Enhanced Solubility and Antimicrobial Activity. Biomolecules 2019, 10, 24. [CrossRef] [PubMed]

23. Hazai, E.; Hazai, I.; Demko, L.; Kovacs, S.; Malik, D.; Akli, P.; Hari, P.; Szeman, J.; Fenyvesi, E.; Benes, E.; et al. Cyclodextrin KnowledgeBase a web-based service managing CD-ligand complexation data. J. Comput. Aided Mol. Des. 2010, 24, 713-717. [CrossRef] [PubMed]

24. Lowe, D.M.; Corbett, P.T.; Murray-Rust, P.; Glen, R.C. Chemical name to structure: OPSIN, an open source solution. J. Chem. Inf. Model. 2011, 51, 739-753. [CrossRef] [PubMed]

25. Chemical Name and Structure Conversion-ChemAxon. Available online: https://chemaxon.com/products/ chemical-name-conversion (accessed on 16 June 2020).

26. Chemical Structure Representation Toolkit-ChemAxon. Available online: https://chemaxon.com/products/ chemical-structure-representation-toolkit (accessed on 16 June 2020).

27. Fourches, D.; Muratov, E.; Tropsha, A. Trust, but verify: On the importance of chemical structure curation in cheminformatics and QSAR modeling research. J. Chem. Inf. Model. 2010, 50, 1189-1204. [CrossRef]

28. Fourches, D.; Muratov, E.; Tropsha, A. Trust, but Verify II: A Practical Guide to Chemogenomics Data Curation. J. Chem. Inf. Model. 2016, 56, 1243-1252. [CrossRef]

29. Moriwaki, H.; Tian, Y.-S.; Kawashita, N.; Takagi, T. Mordred: A molecular descriptor calculator. J. Cheminform. 2018, 10, 4. [CrossRef]

30. Ke, G.; Meng, Q.; Finley, T.; Wang, T.; Chen, W.; Ma, W.; Ye, Q.; Liu, T.-Y. LightGBM: A Highly Efficient Gradient Boosting Decision Tree. In Advances in Neural Information Processing Systems 30; Guyon, I., Luxburg, U.V., Bengio, S., Wallach, H., Fergus, R., Vishwanathan, S., Garnett, R., Eds.; Curran Associates, Inc.: New York, NY, USA, 2017; pp. 3146-3154.

31. Cherkasov, A.; Muratov, E.N.; Fourches, D.; Varnek, A.; Baskin, I.I.; Cronin, M.; Dearden, J.; Gramatica, P.; Martin, Y.C.; Todeschini, R.; et al. QSAR modeling: Where have you been? Where are you going to? J. Med. Chem. 2014, 57, 4977-5010. [CrossRef]

32. Shah, M.; Pore, Y.; Dhawale, S.; Burade, K.; Kuchekar, B. Physicochemical characterization of spray dried ternary micro-complexes of cefuroxime axetil with hydroxypropyl- $\beta$-cyclodextrin. J. Incl. Phenom. Macrocycl. Chem. 2013, 76, 391-401. [CrossRef]

33. Sapte, S.; Pore, Y. Inclusion complexes of cefuroxime axetil with $\beta$-cyclodextrin: Physicochemical characterization, molecular modeling and effect of l-arginine on complexation. J. Pharm. Anal. 2016, 6, 300-306. [CrossRef]

34. Tropsha, A. Best Practices for QSAR Model Development, Validation, and Exploitation. Mol. Inform. 2010, 29, 476-488. [CrossRef]

35. Cysewski, P.; Przybyłek, M. Predicting Value of Binding Constants of Organic Ligands to Beta-Cyclodextrin: Application of MARSplines and Descriptors Encoded in SMILES String. Symmetry 2019, 11, 922. [CrossRef] 
36. Di, P.; Chen, J.; Liu, L.; Li, W.; Tang, Y.; Liu, G. In silico prediction of binding capacity and interaction forces of organic compounds with $\alpha$ - and $\beta$-cyclodextrins. J. Mol. Liq. 2020, 302, 112585. [CrossRef]

37. Salgado, A.; Tatunashvili, E.; Gogolashvili, A.; Chankvetadze, B.; Gago, F. Structural rationale for the chiral separation and migration order reversal of clenpenterol enantiomers in capillary electrophoresis using two different $\beta$-cyclodextrins. Phys. Chem. Chem. Phys. 2017, 19, 27935-27939. [CrossRef] [PubMed]

38. Wei, M.; Yang, X.; Watson, P.; Yang, F.; Liu, H. Development of QSAR model for predicting the inclusion constants of organic chemicals with $\alpha$-cyclodextrin. Environ. Sci. Pollut. Res. Int. 2018, 25, 17565-17574. [CrossRef] [PubMed]

39. Ellouze, F.; Seantier, B.; Amar, N.B.; Deratani, A. Selective separation of $\alpha$ - and $\beta$-cyclodextrins by complexation/ultrafiltration using supra molecular host-guest interaction. Sep. Purif. Technol. 2018, 204, 226-233. [CrossRef]

40. Diamanti, A.C.; Igoumenidis, P.E.; Mourtzinos, I.; Yannakopoulou, K.; Karathanos, V.T. Green extraction of polyphenols from whole pomegranate fruit using cyclodextrins. Food Chem. 2017, 214, 61-66. [CrossRef]

(C) 2020 by the authors. Licensee MDPI, Basel, Switzerland. This article is an open access article distributed under the terms and conditions of the Creative Commons Attribution (CC BY) license (http://creativecommons.org/licenses/by/4.0/). 